Erratum

Surg Radiol Anat (1993) $15: 15-19$

\title{
Anatomic basis of the physiopathology of the epicondylalgias: a study of the deep branch of the radial nerve
}

\author{
AM Rath, M Perez, C Mainguené, AC Masquelet and JP Chevrel
}

This study was done in collaboration with the Laboratoire d'Anatomie, Service de Chirurgie Expérimentale, Bobigny, France and the Service du don des corps du Laboratoire d'Anatomie de l'UFR Biomedicale des Saints Pères, Paris, France.

\section{Announcements}

\section{0th Annual Refresher Course}

The International Skeletal Society

August 18-21, 1993

Toronto, Ontario, Canada

For further information:

The Westin Harbor Castle Hotel

One Harbour Square

Toronto, Ontario, Canada

M5J-1A6

Tel : $1-416-869-1600$

Fax : $1-416-869-1420$

\section{XIXth Congress of the European Society of Neuroradiology \\ 3rd Refresher Course of ESNR \\ Bruges, Belgium, September 8-11, 1993}

Congress main topics:

Neuroradiology in Metabolic and Functional Brain imaging Interventional Neuroradiology

Pediatric Neuroradiology

Advances in PR technology applied to Neuroradiology:

clinical impact, advantages, pitfalls

Socio-economic problems related to Neuroradiology:

education, European concerted studies, optimal algorithms

PACS in Neuroradiology

With the generous support of Schering Diagnostics from Seeing to Understanding

For further information: Congress Secreteriat

ECCO (European Congress Consultants \& Organizers)

rue de l'Abbaye, $27 \mathrm{a}$

B-1050 Brussels, Belgium,

Fax : 32.2. 640.66.97

\section{2nd European Congress of Clinical Anatomy}

\section{Institute of Anatomy, Munich, Germany September 5-7, 1993}

The 2nd European Congress of Clinical Anatomy will be held from Sunday to Tuesday, September 5-7, 1993

For further information, please contact :

Professor Dr. med. Reinhart Putz

Institute of Anatomy

Pettenkoferstraße 11

D-8000 München 2

Germany

Tel : 89.51604810

Fax : 89.51604802

\section{Cours CEPRU-Strasbourg}

Imagerie du tube digestif

Radiographie conventionnelle, échographie,

scanographie, IRM

Strasbourg, France, October 14-15, 1993

Organisé par le Dr MM Filippi de la Palavesa et le Pr JL Dietemann avec la collaboration du Pr C Roy et des Drs A Gangi, F Hannequin, MY Jeung, B Kastler, A Klinkert, P Trotot. A l'institut de Radiologie, 11, rue Humann, F-67085 Strasbourg

Des enseignements théoriques rappellent la sémiologie des principales affections du tube digestif et les indications des différentes méthodes d'imagerie. Une partie importante du temps d'enseignement est consacrée à létude de dossiers intégrant les différentes modalités d'imagerie. Inscriptions limitées à 30 participants

Information: Pr JL Dietemann, Sce. de Radiologie B, Pavillon Clovis Vincent, Hôpitaux Univ./Hôpital Civil, F-67091, Strasbourg cedex, France. Tel: (331) 88161195, Fax: (331) 88161280 